\title{
COMPLEMENT C1Q HAS A NOVEL TETRAMERIC STRUCTURE IN NILE TILAPIA (OREOCHROMIS NILOTICUS)
}

\author{
Meng Chen, Mingmei Ding, Yuhong Wang, Xiaoxue Yin, Shengli Fu, \\ Zheng Guo, Anli Wang, Jianmin Ye $§$
}

\author{
Guangdong Provincial Key Laboratory for Healthy and Safe Aquaculture, \\ Guangdong Engineering Research Center for Environmentally-friendly \\ Aquaculture, College of Life Science, South China Normal University, Guangzhou \\ 510631, People's Republic of China
}

\begin{abstract}
Complement $\mathrm{C} 1 \mathrm{q}$ is the first subcomponent of the classical complement pathway, and a major molecule connecting link between innate and adaptive immunity. Mammalian C1q is a hexamer of a molecular weight about $410 \mathrm{kDa}$; however, little is known about its structural characteristics in teleost. In this study, a rapid method for isolation of the C1q (OnC1q) was developed to investigate its biochemical characterization in Nile tilapia (Oreochromis niloticus). Using a rabbit IgG-Sepharose affinity column, complement OnC1q was isolated from fish sera in a single step, and the C1q further purified by gel filtration through a Sephacryl-S300 column. OnC1q on reduction by electrophoresis on SDS-PAGE yields equimolar amounts of C1qA, C1qB and C1qC chains with molecular weights about 23, 25, $21 \mathrm{kDa}$, respectively. Unreduced OnC1q was shown to be composed of two non-covalently linked subunits of molecular weights about 48 (A-B dimers) and $42 \mathrm{kDa}$ (C-C dimers), with a ratio of 2:1. Further, Gel filtration on Sephacryl-S300 showed that native OnC1q was eluted as a single peak of molecular weight about $280 \mathrm{kDa}$, which was confirmed by SDS-CAGE analysis. Thus, in comparison to the hexameric form in mammals, OnC1q may possess a unique tetrameric structure with a mammalian-like heterotrimeric organization composed of $\mathrm{C} 1 \mathrm{qA}, \mathrm{C1qB}$ and $\mathrm{C} 1 \mathrm{qC}$. The revealing of C1q novel structural in teleost may provide insight into the structural and functional evolutionary history of the $\mathrm{C} 1 \mathrm{q}$ family and the classical pathway in early vertebrates.
\end{abstract}

\section{KEYWORDS}

C1q, Nile tilapia (Oreochromis niloticus), tetrameric structure, evolution

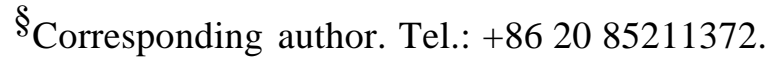

E-mail address: yjmying@126.com 